\title{
Slope-based Empirical Path Loss Prediction Models for Rural Networks at $2.4 \mathrm{GHz}$
}

\author{
${ }^{1}$ Jean Louis Ebongue Kedieng Fendji, ${ }^{2}$ Nelson Maguelva Mafai, ${ }^{3}$ Jean Michel Nlong \\ ${ }^{1}$ Department of Computer Engineering, UIT, University of Ngaoundéré, Cameroon; \\ ${ }^{2}$ Ministry of Scientific Research and Innovation, Yaoundé, Cameroon; \\ ${ }^{3}$ Inter States University, Sangmélima, Cameroon; \\ jlfendji@univ-ndere.cm ; nelsonmafai@gmail.com; jmlong@yahoo.fr
}

\begin{abstract}
Despite the plethora of works on empirical path loss prediction in wireless networks, just a little is addressing rural environments. In this work, we consider slope-based empirical path loss models in wireless networks at $2.4 \mathrm{GHz}$ using off-the-shelf $802.11 \mathrm{n}$ (one transmitter and two receivers at $150 \mathrm{Mbp}$ and $300 \mathrm{Mbps}$ ). We define three scenarios usually observed in rural environment. Subsequently, we do a measurement campaign and compare results to selected prediction models. We later propose a new model based on Liechty model. The new model is compared to Liechty model in Non-Line of Sight (NLOS) and combined (LOS and NLOS) scenarios. The Liechty model provided a better prediction in NLOS scenario while the new model outperforms in combined scenario. In addition, we observe that the data rate also influences the prediction. Especially in free space scenarios, the receiver with the greater data rate provides a smaller mean error and standard deviation.
\end{abstract}

Keywords: Attenuation, measurement, 802.11n, network planning, rural area.

\section{Introduction}

Wireless networks are incontestably an appealing solution to bridge the digital divide between rural and urban regions [1]. This easy-to-deploy technology, especially in hard-to-wire regions or emergency situation [2], can provide bad results and be useless if the network is not well planned [3]. The difficulty when planning a wireless network is to predict the quality of links by estimating the path loss of the signal.

A frequently used tool to predict the quality of signal is the empirical path loss model. However, such a model is usually tied to a specific environment because of the particular configuration. This configuration depends on the devices used as transmitter and the receiver, the distance between them, the frequency of the signal, and the height of the antenna. Despite the great number of path loss models [4], [5], [6], [7], [8], [9], just few are focusing on propagation at $2.4 \mathrm{GHz}$ [10], [11].

However, with the vulgarization of off-the-shelf technologies like IEEE 802.11n, wireless networks at 2.4 $\mathrm{GHz}$ and $5 \mathrm{GHz}$ represent currently an attractive solution to connect rural regions. There is therefore a need to predict the signal path loss at these particular frequencies in rural environment. This is a prior task before estimating the cost of deploying such an infrastructure in rural areas [12]. 
This paper is an extended version of [13]. In this paper, we are interested to provide a more precise empirical model for predicting signal path loss at $2.4 \mathrm{Ghz}$ using off-the-shelf $802.11 \mathrm{n}$ in outdoor environment. For this purpose, we do a measurement campaign in three different scenarios (free space, raised space and built space) using two different receivers (150Mbps and 300Mbps). Afterwards, we compare the gathered data to the attenuation predicted by selected empirical models. Liechty model [11] shows itself as the more precise empirical model by providing the lowest mean error in raised space and built space using both receivers. Finally, we improve this model to obtain a better prediction model by considering the distance to the first breakpoint.

The rest of the paper is organized as follows: section 2 introduces to empirical models and presents the selected models for this study; the methodology, material, scenario and data collection approaches are presented in section 3. Section 4 is dedicated to the numerical analysis of data and section 5 presents and evaluates a new model.

\section{Prediction Models for Wireless Networks}

The prediction of path loss in wireless networks have been tackled by several works. They can be grouped into two classes: theoretical (deterministic) models and empirical ones.

Theoretical models, also called deterministic approaches, are based on the physical phenomena of radio wave propagation. There are different types of theoretical methods: multi-ray models taking into account transmitted and reflected rays [14], [15], and digital or discrete models depending on Maxwell's equations [16], [17]. But in practice, the implementation of deterministic models usually requires a huge knowledge of the field, which is sometimes nearly impossible to obtain in some cases. In addition, deterministic models make use of complex algorithms which usually require a lot of computation depending on the expected accuracy of the model. For this reason, deterministic models are used generally for indoor environments or to well-defined and small size outdoor environments.

A totally different approach for predicting path loss without an exact knowledge of the environment has emerged with the development of statistics and probability. In this approach, the calculation of the signal path loss is made along a single radius shown by the line connecting the transmitter and the receiver. This second class of models is called empirical models.

\subsection{Characteristics of path loss models}

The path loss of a signal is the ratio between the transmitted power and the received power using the following expression (1).

$$
P L=10 \log _{10} \frac{P_{t}}{P_{r}}
$$

Where:

$P L$ : Signal path loss $(d B)$

$P_{t}$ : Transmitted Power $(\mathrm{dBm})$

$P_{r}:$ Received Power $(\mathrm{dBm})$

By using the Friis equation [18], and considering antennas as isotropic, we obtain (2) 


$$
P L_{f s}=20 \log _{10} \frac{(4 \pi d)}{\lambda}
$$

With $c=\lambda f, f$ frequency in $\mathrm{Hz}$ and $c=3.10^{8} \mathrm{~m} \cdot \mathrm{s}^{-1}$, we finally obtain (3) by changing the unit of frequency and the distance

$$
P L_{f s}=\left\{\begin{array}{l}
-92.4+20 \log _{10} d+20 \log 10 f \\
-32.4+20 \log _{10} d+20 \log 10 f
\end{array}\right\}(3.1)
$$

With $d$ in $\mathrm{km}$ and $f$ in $\mathrm{GHz}$ in (3.1) or $\mathrm{MHz}$ in (3.2).

Path loss models are built from the basic model (3). The calculation of signal path loss between the transmitter and the receiver considers in practice other factors such as obstacles and height of antennas.

The list of some common path loss models is given in Table 1.

\begin{tabular}{|c|c|c|}
\hline Models & Condition & Cite \\
\hline \multicolumn{2}{|l|}{ I-Free space } & {$[18]$} \\
\hline |-Egli & $f \in] 30 ; 3000[$ & [4] \\
\hline \multicolumn{2}{|l|}{ |-One Slope } & {$[19]$} \\
\hline \multicolumn{2}{|l|}{ |-Dual Slope } & {$[19]$} \\
\hline \multicolumn{2}{|l|}{ |-Log Normal Shadowing } & {$[20]$} \\
\hline \multicolumn{2}{|l|}{ |-Partitioned } & {$[20]$} \\
\hline |-Liechty & $f \approx 2400$ & {$[11]$} \\
\hline I-Okumura & $f \in] 150 ; 1920[; d \in] 1 ; 100\left[; h_{t x} \in\right] 30 ; 200\left[; h_{r x} \in\right] 3 ; 10[$ & [5] \\
\hline |-Okumura-Hata & $f \in] 150 ; 1500[; d \in] 1 ; 10\left[; h_{t x} \in\right] 30 ; 200\left[; h_{r x} \in\right] 1 ; 10[$ & [6] \\
\hline |-COST 231 Hata & $f \in] 150 ; 2000[; d \in] 1 ; 20\left[; h_{t x} \in\right] 30 ; 200\left[; h_{r x} \in\right] 1 ; 10[$ & {$[21]$} \\
\hline |-Hata-Davidson & $f \in] 150 ; 1500[; d \in] 1 ; 300\left[; h_{t x} \in\right] 30 ; 200\left[; h_{r x} \in\right] 1 ; 10[$ & {$[24]$} \\
\hline |-Rural & $f \in\{160 ; 400 ; 900\}$ & [8] \\
\hline |-ITU-R & $1.5<f<2 ; 1<d<10 ; 30<h_{t x}<200 ; 1<h_{r x}<10$ & {$[26]$} \\
\hline I-ECC-33 & $700 \leq f \leq 3500 ; 1<d<10 ; 20<h t x<200 ; 1<h_{r x}<10$ & {$[25]$} \\
\hline |-Green Obaidat & & {$[22]$} \\
\hline |-Erceg & $f \approx 2000 ; 1<h_{r x}<2$ & [7] \\
\hline |-SU| & $2500<f<2700 ; 0.1<d<8 ; 20<h_{t x}<80 ; 2<h_{r x}<10$ & {$[23]$} \\
\hline $\begin{array}{l}f: \text { frequency }(\mathrm{MHz}) ; \quad d: \text { dis } \\
h_{t x} \text { : height of transmitter an }\end{array}$ & $\begin{array}{l}\text { tween the transmitter and the receiver }(\mathrm{Km}) \\
\mathrm{n)} ; h_{r x} \text { : height of the receiver antenna }(\mathrm{m})\end{array}$ & \\
\hline
\end{tabular}

Table 1. Common empirical path loss models 


\subsection{Selected models}

The present study focuses on five prediction models generally used in micro-cellular areas: One Slope, Dual Slope, Log Normal Shadowing, Partitioned, and Liechty.

\subsubsection{One Slope Model}

The One Slope model is a Log Distance based prediction model. In [18], authors show how the attenuation of the signal weakens with the distance. The attenuation on an exponent which indicates how the path loss increases rapidly with distance. The expression of the path loss is given by (4).

$$
P L_{1 \text { Slope }}=P L_{f s}\left(d_{0}\right)+10 n \log _{10}\left(\frac{d}{d_{0}}\right)
$$

Where:

$P L_{f s}\left(d_{0}\right):$ Signal path loss in free space at distance $d_{0}(m)$

$d_{0}$ : Distance of reference $(\mathrm{m})$

$d$ : Distance between the transmitter and the receiver $(\mathrm{m})$

$n$ : Path loss exponent of the environment

The expression of $P L_{f s}\left(d_{0}\right)$ is given by (3). Prior measurements are required in order to calibrate the parameter $n$ by using the least squares method. An obvious limit of this model is that it does not consider obstacles between the transmitter and the receiver.

\subsubsection{Dual-slope Model}

The Dual-slope model extends the One Slope model [18]. The first slope considers Line Of Sight (LOS), and the second slope considers Non-Line Of Sight (NLOS). The expression of the path loss is given by (5).

$$
P L_{2 \text { Slope }}=P L_{f s}\left(d_{0}\right)+\left\{\begin{array}{c}
10 n_{1} \log _{10} d \\
10 n_{1} \log _{10} d_{b p}+10 n_{2} \log _{10} \frac{d}{d_{b p}}
\end{array}\right\}
$$

With $1 m<d \leq d_{b p}$ in (5.1) and $d>d_{b p}$ in (5.2).

Where:

$P L_{f s}\left(d_{0}\right)$ : Signal path loss in free space at distance $d_{0}(d B)$

$d_{0}$ : Distance of reference $(\mathrm{m})$

$d$ : Distance between the transmitter and the receiver $(\mathrm{m})$

$d_{b p}$ : Distance between the transmitter and the first obstacle $(\mathrm{m})$

$n_{1}$ : Path loss exponent of the environment $d \leq d_{b p}$ 
$n_{2}$ : Path loss exponent of the environment $d>d_{b p}$

The expression of $P L_{f s}\left(d_{0}\right)$ is still given by (3) and values of the exponents $n_{1}$ and $n_{2}$ are determined using the least squares method and field measurements.

\subsubsection{Log Normal Shadowing Model}

The Log Normal Shadowing Model is another improvement of One Slope model [18]. It considers that the signal path loss at a distance is in fact a random variable, due to the effect of multipath propagation and obstacles encountered by the wave. The expression of the path loss is given by (6).

$$
P L_{L N-S h a}=P L_{f s}\left(d_{0}\right)+10 n \log _{10}\left(\frac{d}{d_{0}}\right)+\chi_{\sigma}
$$

Where:

$P L_{f s}\left(d_{0}\right):$ Signal path loss in free space at distance $d_{0}(d B)$

$d_{0}$ : Distance of reference $(\mathrm{m})$

$d$ : Distance between the transmitter and the receiver $(\mathrm{m})$

$\chi_{\sigma}$ : Shadowing effect $(\mathrm{dB})$

$n$ : Path loss exponent of the environment

The expression of $P L_{f s}\left(d_{0}\right)$ is still given by (3). $\chi_{\sigma}$ is a Gaussian random variable (in $\mathrm{dB}$ ) with zero mean and standard deviation $\sigma$. Values of $\mathrm{n}$ and $\sigma$ are also determined from field measurements [18].

\subsubsection{Partitioned Model}

This model is also an extension of the One Slope model. It has four different expressions of signal path loss depending on the distance between the transmitter and the receiver. The expression of the path loss is given by (7) [24].

$$
P L_{\text {part }}=P L_{f s}\left(d_{0}\right)+\left\{\begin{array}{c}
20 \log _{10} d \\
20+30 \log _{10} \frac{d}{10} \\
29+60 \log _{10} \frac{d}{20} \\
47+120 \log _{10} \frac{d}{40}
\end{array}\right\} \begin{aligned}
& (7.1) \\
& (7.2) \\
& (7.4)
\end{aligned}
$$

With $1 m<d \leq 10 m$ in (7.1), $10 m<d \leq 20 m$ in (7.2), $20 m<d \leq 40 m$ in (7.3) and $d>40 m$ in (7.4). Like in previous models, the expression of $P L_{f s}\left(d_{0}\right)$ is also given by (3). 


\subsubsection{Liechty Model}

The Liechty Model has been proposed by Christopher Lorne Liechty [11]. The model extends the One slope model in order to consider the attenuation due to obstacles such as trees and buildings. The expression of the path loss is given by (8).

$$
P L_{\text {Liechty }}=P L_{f s}\left(d_{0}\right)+10 n \log _{10}\left(\frac{d}{d_{0}}\right)+\sum_{i} n u m_{i} * a_{i}
$$

Where:

$P L_{f s}\left(d_{0}\right):$ Signal path loss in free space at distance $d_{0}(m)$

$d_{0}:$ Distance of reference $(\mathrm{m})$

$d$ : Distance between the transmitter and the receiver $(\mathrm{m})$

num $_{i}:$ Number of obstructions of type $\mathrm{i}$

$a_{i}$ : Attenuation of obstructions of type i (dB)

$n$ : Path loss exponent of the environment

The expression of $P L_{f s}\left(d_{0}\right)$ is given by (3), as it has been the case in previous models.

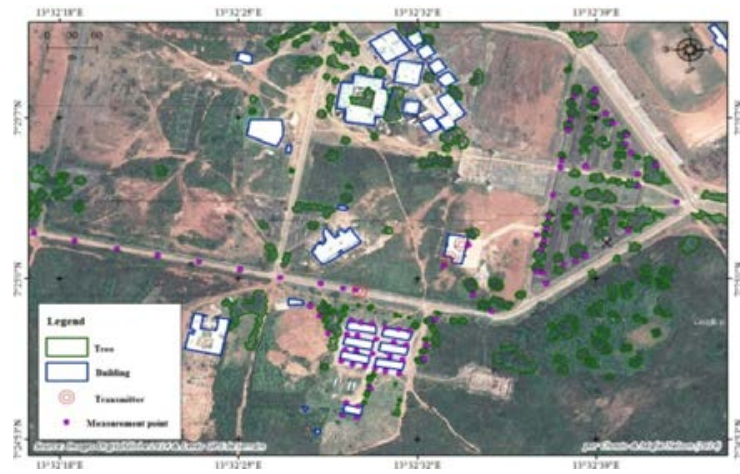

(a)

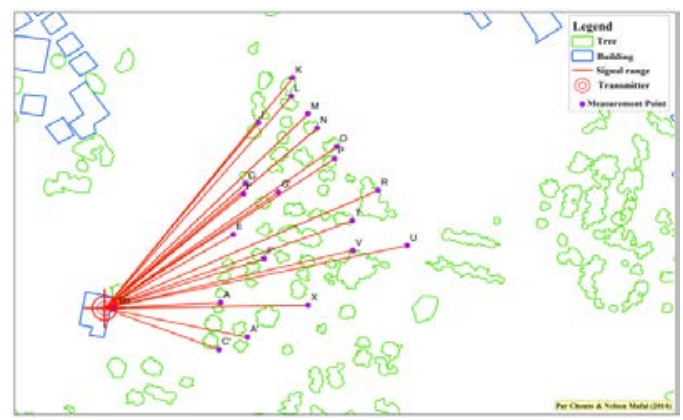

(c)

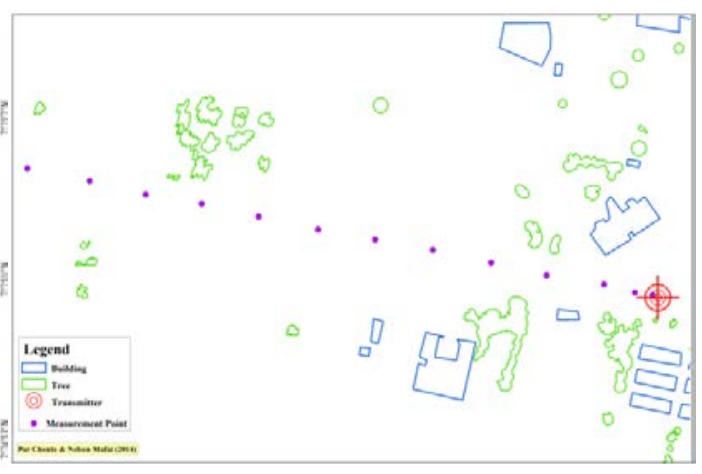

(b)

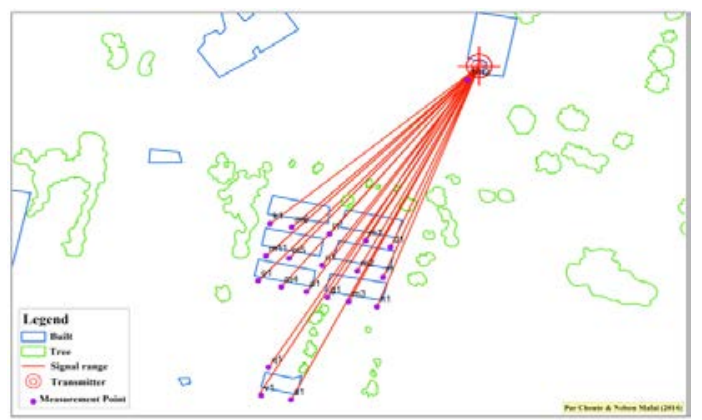

(d)

Figure 1. Measurement points on the field. 
Jean Louis Ebongue Kedieng Fendji, Nelson Maguelva Mafai, Jean Michel Nlong; Slope-based Empirical Path Loss

Prediction Models for Rural Networks at $2.4 \mathrm{GHz}$, Transactions on Networks and Communications, Volume 7 No.

1, February (2019); pp: 84-101

\section{Methodology}

\subsection{Environment of the study}

The selected area for measurements is the campus of the University of Ngaoundere, in Cameroon. This area is mainly characterised by randomly distributed trees and some small buildings as shown in Figure 1.a. To carry out measurements, three scenarios have been designed:

- Free space: a distance of $600 \mathrm{~m}$ has been selected. At each interval of $50 \mathrm{metres}$, the signal strength is measured. Figure 1.b shows the different points of measurement.

- Wooded area: the average height of trees is around 6,5metres. Fig.1.c shows the different points of measurement for this scenario.

Built-up area: houses have an average height of about 3.5metres. The different points of measurements are shown in Figure 1.d.

\subsection{Description of the hardware and software tools}

The access point used as a transmitter in all scenarios is manufactured by Alpha Network and complies with the IEEE 802.11n standard. it offers a bandwidth of $150 \mathrm{Mbps}$ and operates at $2.4 \mathrm{GHz}$. The transmit power is $30 \mathrm{dBm}$ with an antenna gain of $12 \mathrm{dBi}$. Two wireless USB dongles are used as receivers. Both are compliant to the IEEE $802.11 \mathrm{n}, 802.11 \mathrm{~b}$ and $802.11 \mathrm{~g}$ standards. They are operating at $2.4 \mathrm{GHz}$ but providing different bandwidth (150Mbps and 300Mbps). A USB GPS is used to determine the different points for measurements. The characteristics of hardware is provided in Table 2.

We used the free and open source software Vistumbler in version 9.8 for wireless signal strength measurement. It enables live Google Earth tracking of access points and supports GPS integration.

Table 2. Characteristics of hardware

\begin{tabular}{|l|l|}
\hline \multicolumn{1}{|c|}{ Type of equipment } & \multicolumn{1}{c|}{ Characteristics } \\
\hline Access Point & Manufacturer: Alpha Network, Model: N2, Power: $30 \mathrm{dBm}$, Antenna: $12 \mathrm{dBi}$ \\
\hline Wireless USB dongle 1 & $\begin{array}{l}\text { Manufacturer: Dodocool, Rate: } 300 \mathrm{Mbps,} \text { Chipset: Realtek 8191, Driver: } \\
\text { RTL8188SU/8191FEB28, Antenna: } 2 \mathrm{dBi}\end{array}$ \\
\hline Wireless USB dongle 2 & $\begin{array}{l}\text { Manufacturer: Dodocool, Rate: } 150 \mathrm{Mbps,} \text { Chipset: Ralink RT5370, Driver: } \\
\text { RALINK23FEB, Antenna: } 2 \mathrm{dBi}\end{array}$ \\
\hline USB GPS & Manufacturer: Navilock, Model: NL-464US 60122, Sensibility: $-159, \mathrm{dBm}$ \\
\hline
\end{tabular}

\subsection{Data collection and analysis}

We used the Single Marker Measurements [11] as positioning mode. The antennae of the USB dongles are oriented towards the sky throughout the measurement campaign. Ten measurements are performed at each selected point with the help of Vistumbler. Retrieved data are recorded in predefined Datasheets. Data analysis is performed in five steps. First, we calculate the mean loss of signal. Second, we determine the fitting curve by using the least squares method. Third, we evaluate the path loss exponent $n$. Fourth, 
we calculate the $\sigma$ parameter of Log Normal Shadowing Model. Finally, we compare the predicted by the selected models with the mean loss of the signal measured in the field.

The path loss exponent is determined using the least squares method. The attenuation of each type of obstacle required by the Liechty model is considered as the difference between the mean loss of the signal obtained before and after the obstacle.

The value of $\sigma$ is determined using (9):

$$
\sigma^{2}=\frac{\sum_{i}\left(\overline{P_{m}}-\overline{\left.P_{r}\right)^{2}}\right.}{k} 1 \leq i \leq k
$$

With:

$\overline{P_{m}}:$ Power of the measured received signal

$\overline{P_{r}}:$ Strength of the estimated received signal

$k:$ Number of measurement points

\section{Results interpretation}

Table 3 provides empirical values of the model parameters. The mean error and the standard deviation are used as indicators to compare the predicted values to the measured ones.

Table 3. Empirical values of the model parameters

\begin{tabular}{|l|l|l|c|}
\hline \multicolumn{2}{|l|}{} & \multicolumn{2}{c|}{ 300Mbps / 150Mbps } \\
\hline Environment & Obstruction (dB) & $n$ & $\sigma$ \\
\hline Free Space & 0 & $1.950 / 2.186$ & $1.12 / 1.50$ \\
\hline Wooded area & 0.37 & $3.407 / 3.656$ & $2.41 / 2.61$ \\
\hline Built-up area & 0.41 & $4.799 / 4.996$ & $2.10 / 2.48$ \\
\hline
\end{tabular}

\subsection{Result analysis in free spaces}

A total number of 100 measurements have been performed in free space. Figure 2 shows the pathloss of the signal for both USB wireless receivers (300Mbps and $150 \mathrm{Mbps}$ ) depending on the distance Transmitter - Receiver (TR). Obviously, the mean loss of the signal increases as the TR distance increases. However, the $300 \mathrm{Mbps}$ wireless USB receiver provides lower losses than the $150 \mathrm{Mbps}$ one. Consequently, the maximal distance at which the signal is still useful $(90 \mathrm{~dB})$ is about 350metres and 320metres for respectively $300 \mathrm{Mbps}$ and $150 \mathrm{Mbps}$ receiver. 


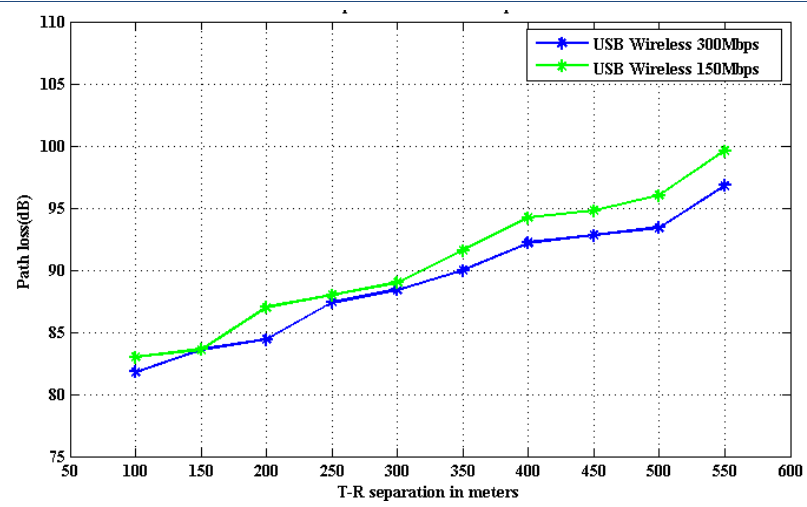

Fig. 2. Mean losses in free space

Comparison between models is summarised in Table 4. From Figure 3, One Slope, Dual Slope and Liechty models are quite close to the measured values. Because of their large errors, results from the Partitioned model could not be plotted.

From the experimental results provided in Table 4, One Slope, Dual Slope, and Liechty models are the best in free space. In fact, they provide a mean error of $0.90 \mathrm{~dB}$ and a standard deviation of $1.12 \mathrm{~dB}$ for the $300 \mathrm{Mbps}$ receiver, and a mean error of $1.12 \mathrm{~dB}$ and a standard deviation of $1.50 \mathrm{~dB}$ for the $150 \mathrm{Mbps}$ receiver.

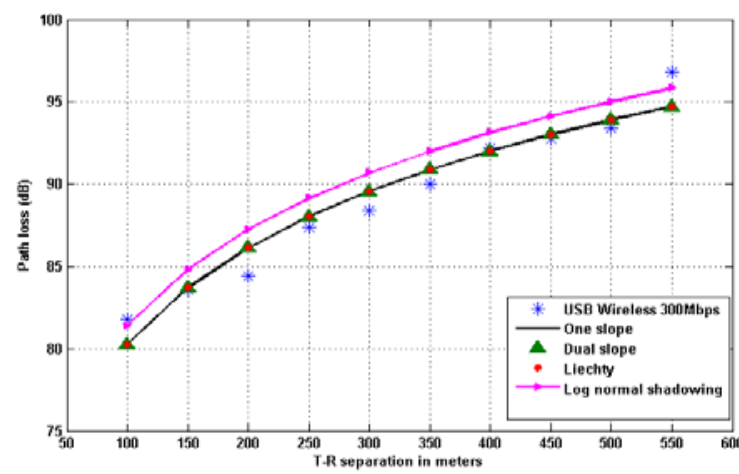

a) $300 \mathrm{Mbps}$ wireless receiver

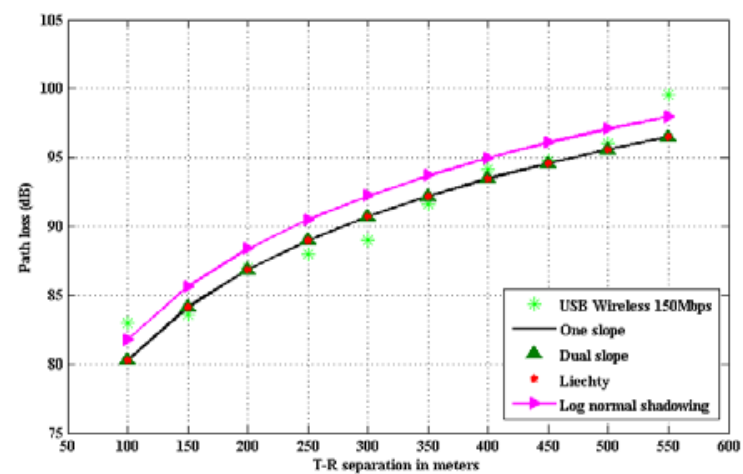

b) $150 \mathrm{Mbps}$ wireless receiver

Fig. 3. Comparison of the predicted values in free space.e 
Table 4. Experimental results in free space

\begin{tabular}{lll}
\hline \multirow{2}{*}{ Models } & \multicolumn{2}{l}{ 300Mbps / 150Mbps } \\
\cline { 2 - 3 } & Mean Error (dB) & Standard Deviation (dB) \\
\hline One Slope & $0.90 / 1.12$ & $1.12 / 1.50$ \\
Dual Slope & $0.90 / 1.12$ & $1.12 / 1.50$ \\
Lietchy & $0.90 / 1.12$ & $1.12 / 1.50$ \\
Log Normal & $1.54 / 1.71$ & $1.68 / 1.85$ \\
Partitioned & $141.03 / 103.83$ & $142.89 / 104.06$ \\
\hline
\end{tabular}

\subsection{Result analysis in a wooded area}

A total number of 200 measurements have been performed in wooded area. Figure 4 shows the pathloss of the signal for both USB wireless receivers (300Mbps and 150Mbps) depending on the TR distance. Figure 4 shows an increasing saw tooth curve, in contrary to the fairly linear curve observed in free space. The main reason is the presence of obstacles which do not ease the prediction of the path loss at any point. However, as it is the case in free space, the $300 \mathrm{Mbps}$ wireless USB receiver provides lower losses than the $150 \mathrm{Mbps}$ one. In addition, the maximal distance at which the signal is still useful $(90 \mathrm{~dB})$ is about 182 metres and 177 metres for respectively $300 \mathrm{Mbps}$ and $150 \mathrm{Mbps}$ receiver.

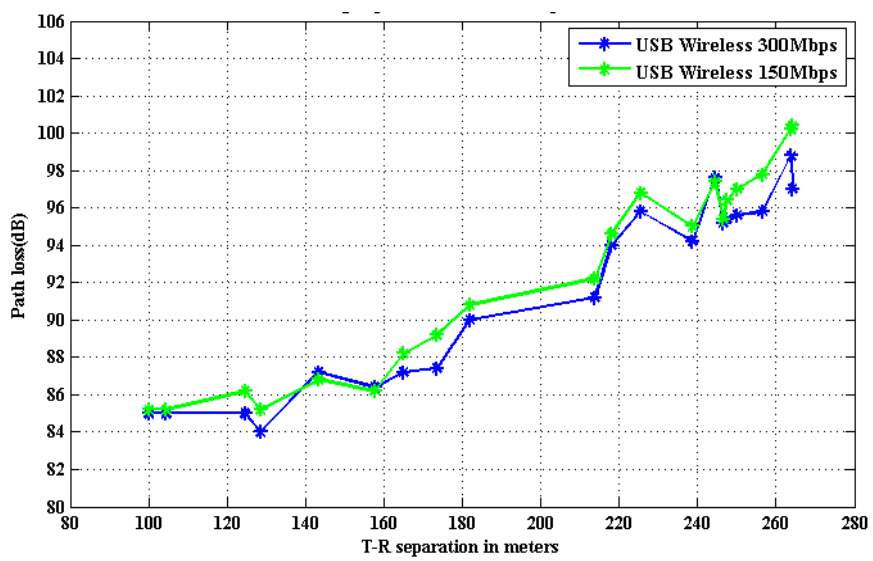

Fig. 4. Mean losses in wooded area.

From Figure 5, the predicted values from the Liechty model are quite close to the measured ones. 


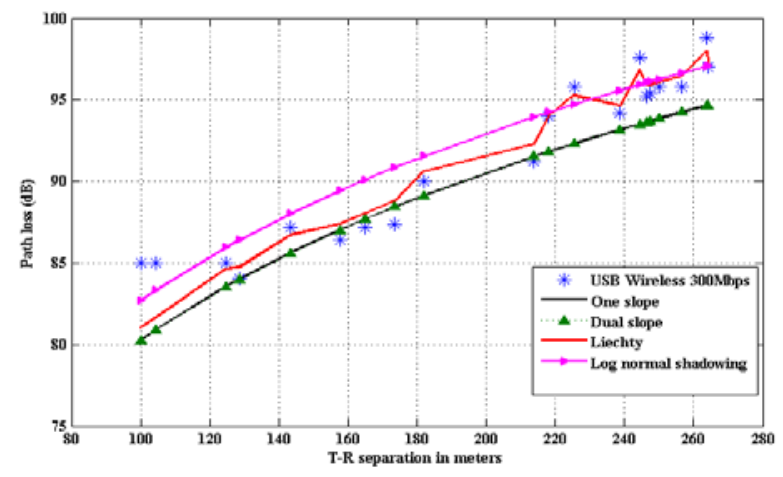

a) $300 \mathrm{Mbps}$ wireless receiver

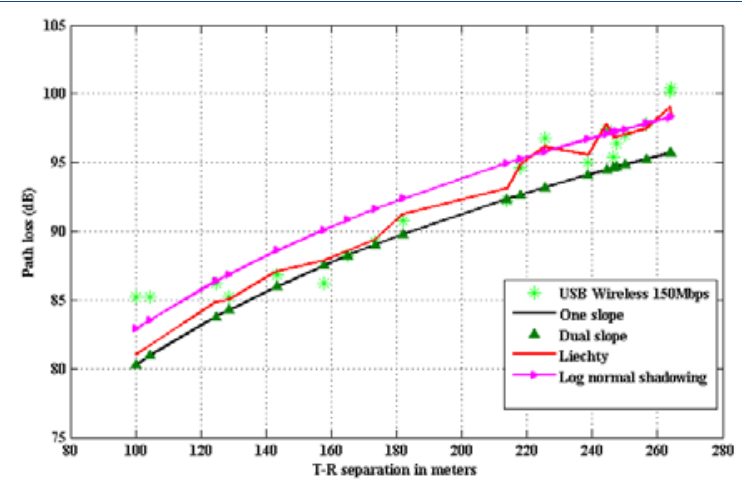

b) $150 \mathrm{Mbps}$ wireless receiver

Fig. 5. Comparison of the predicted values in the wooded area.

According to the experimental results provided in Table 5, the Liechty model provides the best results. In fact, this model provides a mean error of $0.94 \mathrm{~dB}$ and a standard deviation of $1.35 \mathrm{~dB}$ for the $300 \mathrm{Mbps}$ receiver; and a mean error of $1.04 \mathrm{~dB}$ and a standard deviation of $1.50 \mathrm{~dB}$ for the $150 \mathrm{Mbps}$ receiver.

Table 5. Experimental results in wooded area

\begin{tabular}{lll}
\hline \multirow{2}{*}{ Models } & \multicolumn{2}{l}{ 300Mbps / 150Mbps } \\
\cline { 2 - 3 } & Mean Error $(\mathrm{dB})$ & Standard Deviation $(\mathrm{dB})$ \\
\hline One Slope & $1.73 / 2.02$ & $2.10 / 2.48$ \\
Dual Slope & $1.73 / 2.02$ & $2.10 / 2.48$ \\
Lietchy & $1.60 / 1.27$ & $2.03 / 1.78$ \\
Log Normal & $1.97 / 1.85$ & $2.49 / 2.30$ \\
Partitioned & $109.71 / 108.48$ & $109.93 / 108.69$ \\
\hline
\end{tabular}

\subsection{Result analysis in built-up areas}

A total number of 200 measurements have been performed in built-up area. Figure 6 shows the pathloss of the signal for both USB wireless receivers (300Mbps and 150Mbps) depending on the TR distance. Because of the presence of obstacles, Figure 6 also shows a non-monotonic increasing curve. As expected, since it is the case in free space and wooded area, the 300Mbps wireless USB receiver provides lower losses than the $150 \mathrm{Mbps}$ one. From Figure 7, the predicted values from the Liechty model are quite close to the measured ones. In contrary to previous scenarios, the maximal distance at which the signal is still useful $(90 \mathrm{~dB})$ is the same and about 165 metres, irrespectively the type of receiver. 


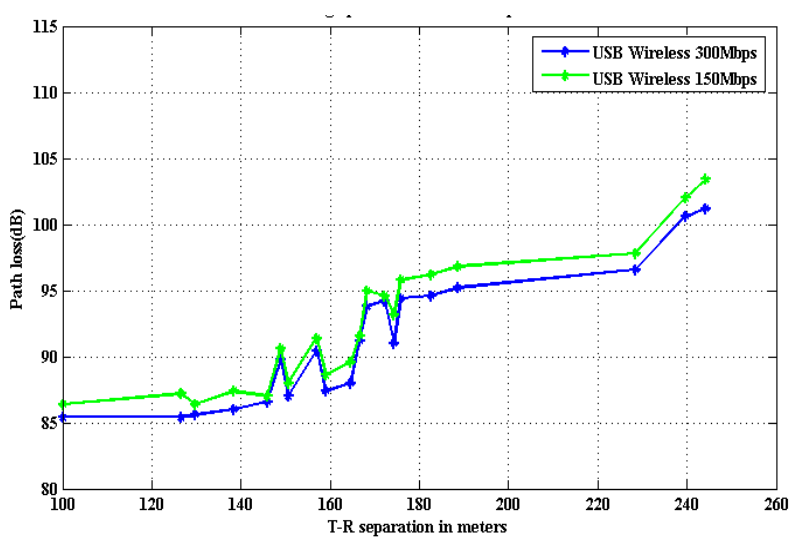

Fig. 6. Mean losses in a built-up area.

Table 6. Experimental results in built-up area

\begin{tabular}{lll}
\hline \multirow{2}{*}{ Models } & \multicolumn{2}{l}{$300 \mathrm{Mbps} /$ 150Mbps } \\
\cline { 2 - 3 } & Mean Error (dB) & Standard Deviation (dB) \\
\hline One Slope & $1.73 / 2.02$ & $2.10 / 2.48$ \\
Dual Slope & $1.73 / 2.02$ & $2.10 / 2.48$ \\
Lietchy & $1.60 / 1.27$ & $2.03 / 1.78$ \\
Log Normal & $1.97 / 1.85$ & $2.49 / 2.30$ \\
Partitioned & $109.71 / 108.48$ & $109.93 / 108.69$ \\
\hline
\end{tabular}

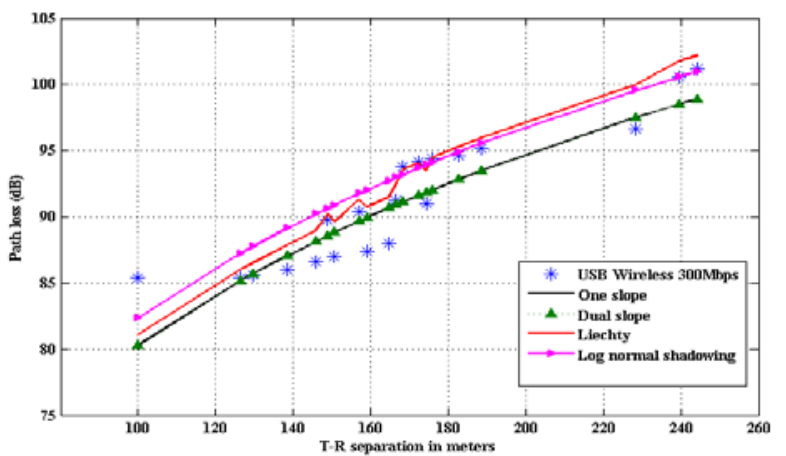

a) $300 \mathrm{Mbps}$ wireless receiver

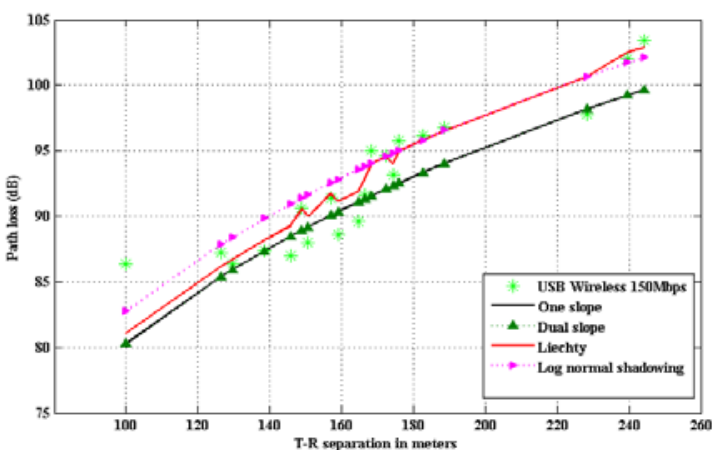

b) $150 \mathrm{Mbps}$ wireless receiver

Fig. 7. Comparison of predicted values in a built-up area.

From the experimental results provided in Table 6, the Liechty model provides once more the best results. Indeed, this model provides a mean error of $1.60 \mathrm{~dB}$ and a standard deviation of $2.03 \mathrm{~dB}$ for the $300 \mathrm{Mbps}$ receiver; and a mean error of $1.27 \mathrm{~dB}$ and a standard deviation of $1.78 \mathrm{~dB}$ for the other one. new model.

\section{Improving the Lietchy model in combined environment}

The better results of the Liechty model are justified by the fact that the model considers the number of obstacles between transmitter and receiver as well as their attenuation on the signal. However, we assumed that the distance between the transmitter and each attenuation point may also influence the 
Jean Louis Ebongue Kedieng Fendji, Nelson Maguelva Mafai, Jean Michel Nlong; Slope-based Empirical Path Loss Prediction Models for Rural Networks at $2.4 \mathrm{GHz}$, Transactions on Networks and Communications, Volume 7 No. 1, February (2019); pp: 84-101

quality of the signal. Therefore, considering those distances during the prediction of the signal could improve the accuracy. But, since it is difficult to consider the distances of all those attenuation points, we take only into account the distance to the first obstacle. This point is called the breakpoint. We suppose that the greatest attenuation of the signal occurs at this point.

\subsection{Path loss expressions}

The new model is obtained by merging the parameters of the Dual slope and the Liechty models. We obtain equation (10):

$$
P L_{\text {new }}=P L_{f s}\left(d_{0}\right)+\left\{\begin{array}{c}
10 n_{1} \log _{10} d \\
10 n_{2} \log _{10} \frac{d}{d_{b p}}+10 n_{1} \log _{10} d_{b p}+\sum_{i} n u m_{i} * a_{i}
\end{array}\right\}
$$

With, $1 m<d \leq d_{b p}$ in (10.1) and $d>d_{b p}$ in (10.2).

Where:

$P L_{f s}\left(d_{0}\right):$ Signal path loss in free space at distance $\mathrm{d}_{0}(\mathrm{~m})$

$d_{0}$ : Distance of reference $(\mathrm{m})$

$d$ : Distance between the transmitter and the receiver $(\mathrm{m})$

$d_{b p}$ : Distance from the transmitter to the first obstacle $(\mathrm{m})$

num $_{i}$ : Number of obstructions of type i

$a_{i}$ : Attenuation of obstructions of type i (dB)

$n_{1}$ : Path loss exponent of the environment $d \leq d_{b p}$

$n_{2}$ : Path loss exponent of the environment $d>d_{b p}$

\subsection{Accuracy analysis of the model}

The accuracy of the new model is analyzed by considering the measurements of the signal in both LOS and NLOS. Only wooded and built-up areas are considered. The consideration of both LOS and NLOS is materialized by using different path loss exponents, as it is the case in the new model: $n_{1}$ in LOS and $n_{2}$ in NLOS. The accuracy is evaluated in NLOS environment and in combined environment (LOS and NLOS) environment.

\subsubsection{Wooded area}

Table 7 presents the results of the accuracy analysis in the wooded area. From Table 7, even if both models provide similar results, Liechty model is more precise in NLOS environment. However, the new model provides better predictions in combined environment. In fact, the new model provides in combined environment a mean error of $2.33 \mathrm{~dB}$ and $2.67 \mathrm{~dB}$ respectively for $300 \mathrm{Mbps}$ and $150 \mathrm{Mbps}$ USB receivers; 
which are lower compared to $4.22 \mathrm{~dB}$ and $4.00 \mathrm{~dB}$ provided by Liechty model. Likewise, the new model also provided in combined environment a standard deviation of $4.02 \mathrm{~dB}$ and $4.21 \mathrm{~dB}$ respectively for $300 \mathrm{Mbps}$ and $150 \mathrm{Mbps}$ receivers; which are also lower compared to $5.12 \mathrm{~dB}$ and $5.09 \mathrm{~dB}$ provided by Liechty model. Figure 8 and 9 give a graphical comparison between both models and the measured data in wooded area, respectively for combined (LOS and NLOS) and NLOS environments.

Table 7. Results of the accuracy analysis in the wooded area

\begin{tabular}{|c|c|c|c|c|}
\hline \multirow{2}{*}{ Models } & \multicolumn{2}{|c|}{ LOS and NLOS (300Mbps/150Mbps) } & \multicolumn{2}{|c|}{ NLOS only (300Mbps/150Mbps) } \\
\hline & Mean Error (dB) & Std Deviation (dB) & Mean Error (dB) & Std Deviation (dB) \\
\hline Liechty & $4.22 / 4.00$ & $5.12 / 5.09$ & $0.94 / 1.04$ & $1.35 / 1.50$ \\
\hline New model & $2.33 / 2.67$ & 4.02 / 4.21 & $1.13 / 2.02$ & 1.73 / 2.41 \\
\hline
\end{tabular}

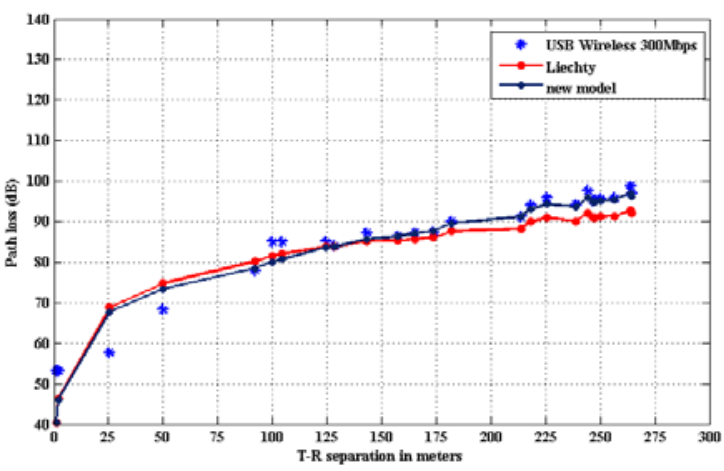

a) $300 \mathrm{Mbps}$ wireless receiver

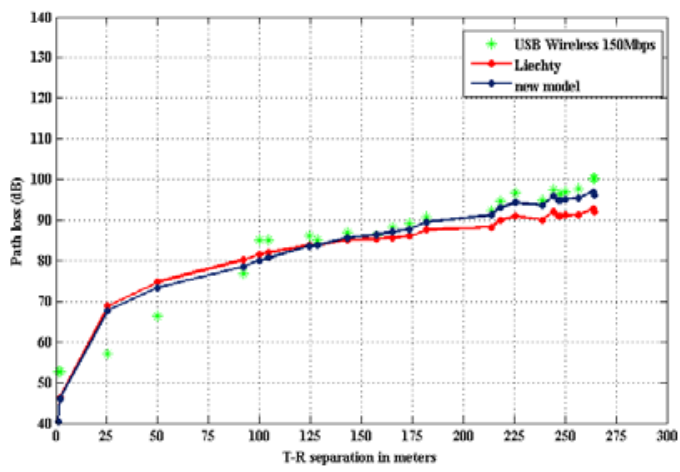

b) $150 \mathrm{Mbps}$ wireless receiver

Fig. 8. Accuracy of models in the wooded area (combined: LOS and NLOS).

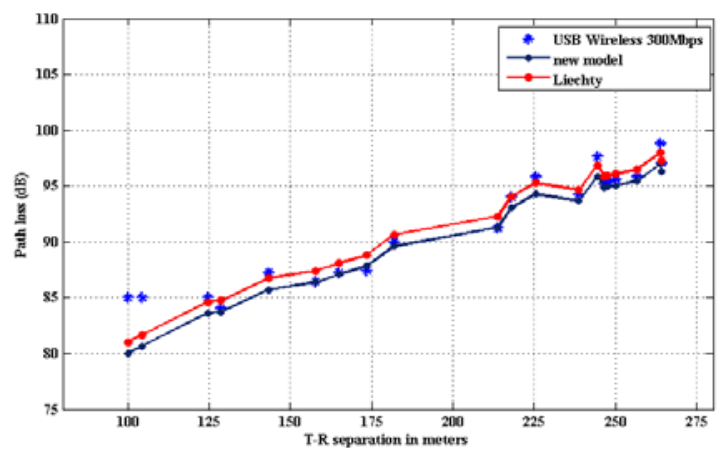

a) $300 \mathrm{Mbps}$ wireless receiver

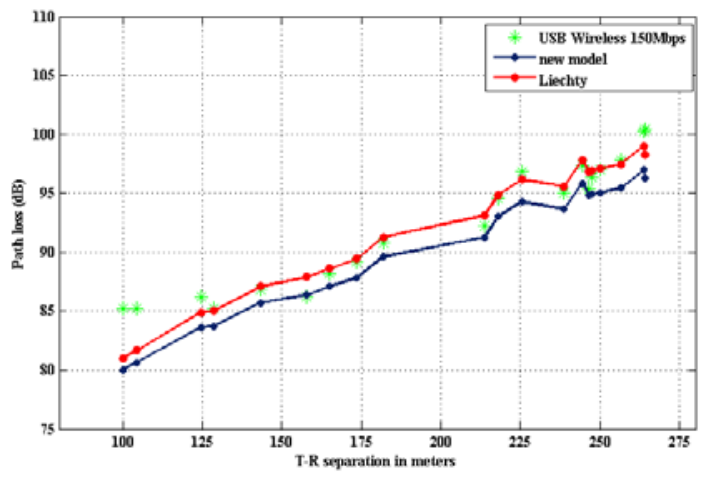

b) $150 \mathrm{Mbps}$ wireless receiver

Fig. 9. Accuracy of models in the wooded area (NLOS).

\subsubsection{Built-up area}

Table 8 presents the results of the accuracy analysis in the built-up area. From Table 8, even if both models provide similar results, Liechty model provides better predictions in NLOS environment. But, the new model is more precise in combined environment as it was the case in wooded area. Actually, the new 
Jean Louis Ebongue Kedieng Fendji, Nelson Maguelva Mafai, Jean Michel Nlong; Slope-based Empirical Path Loss Prediction Models for Rural Networks at $2.4 \mathrm{GHz}$, Transactions on Networks and Communications, Volume 7 No. 1, February (2019); pp: 84-101

model provides in combined environment a mean error of $2.36 \mathrm{~dB}$ and $2.61 \mathrm{~dB}$ respectively for $300 \mathrm{Mbps}$ and $150 \mathrm{Mbps}$ receivers; which are low compared to $4.35 \mathrm{~dB}$ and $4.00 \mathrm{~dB}$ provided by Liechty model. Likewise, the new model also provided in combined environment a standard deviation of $4.01 \mathrm{~dB}$ and $4.19 \mathrm{~dB}$ respectively for $300 \mathrm{Mbps}$ and $150 \mathrm{Mbps}$ receivers; which are also low compared to $5.46 \mathrm{~dB}$ and $5.45 \mathrm{~dB}$ provided by Liechty model. Figure 10 and 11 give a graphical comparison between both models and the measured data in built-up area, respectively for combined (LOS and NLOS) and NLOS environments.

Table 8. Results of the accuracy analysis in the wooded area (LOS and NLOS)

\begin{tabular}{lcccc}
\hline \multirow{2}{*}{ Models } & \multicolumn{2}{c}{ LOS and NLOS (300Mbps/150Mbps) } & \multicolumn{2}{c}{ NLOS only (300Mbps/150Mbps) } \\
\cline { 2 - 4 } & Mean Error (dB) & Std Deviation (dB) & Mean Error (dB) & Std Deviation (dB) \\
\hline Liechty & $4.35 / 4.00$ & $5.46 / 5.45$ & $1.60 / 1.27$ & $2.03 / 1.78$ \\
New model & $2.36 / 2.61$ & $4.01 / 4.19$ & $1.73 / 1.41$ & $2.15 / 1.88$ \\
\hline
\end{tabular}

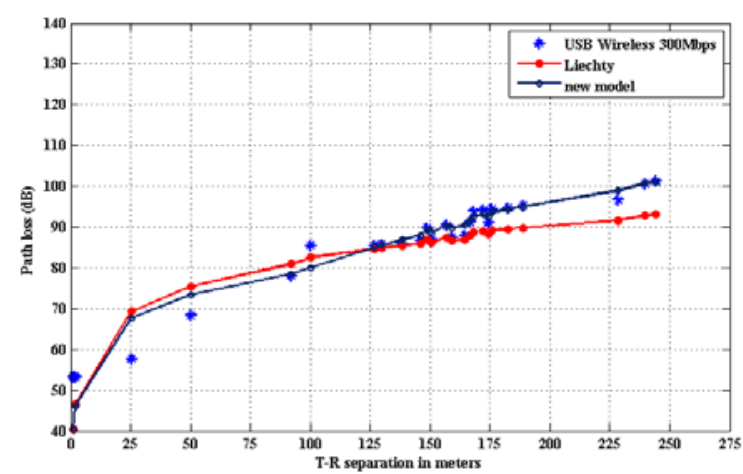

a) $300 \mathrm{Mbps}$ wireless receiver

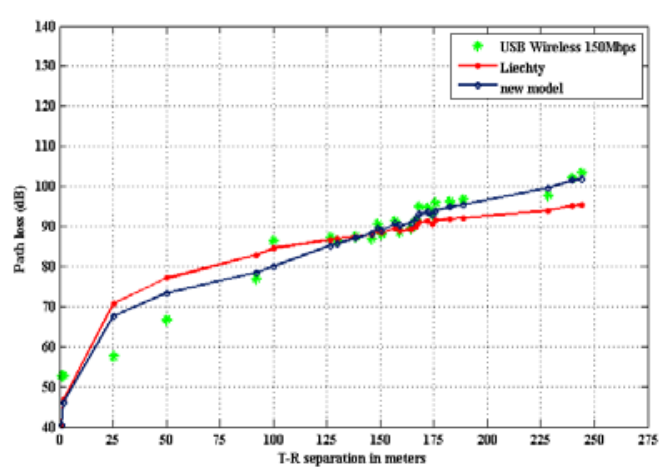

b) $150 \mathrm{Mbps}$ wireless receiver

Fig. 10. Accuracy of models in the built-up area (combined: LOS and NLOS).

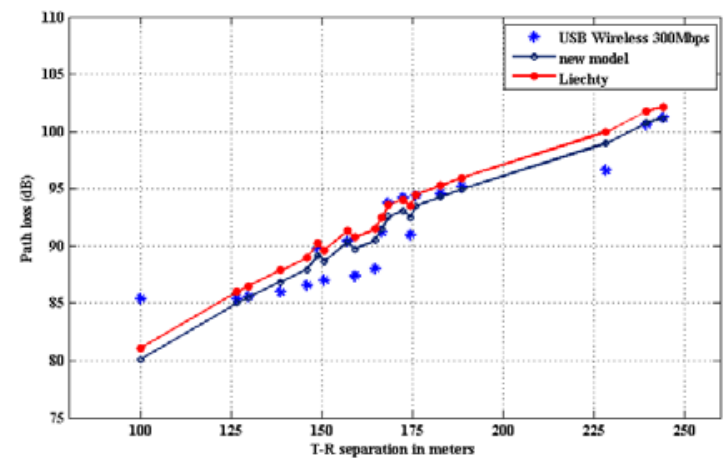

a) $300 \mathrm{Mbps}$ wireless receiver

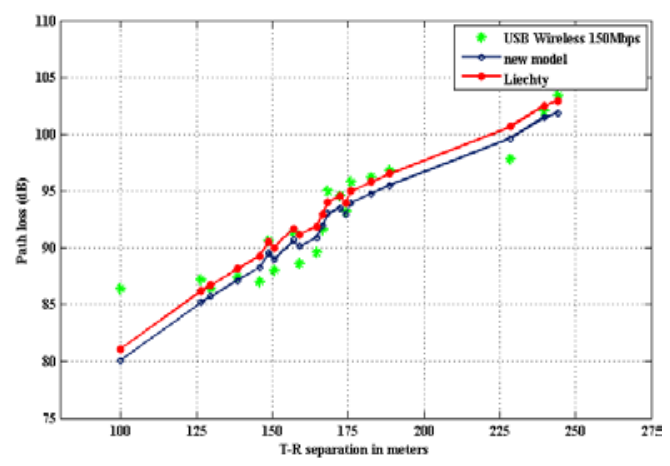

b) $150 \mathrm{Mbps}$ wireless receiver

Fig. 11. Accuracy of models in the built-up area (NLOS). 


\section{Conclusion}

The aim of this work was to determine a precise empirical path loss model for rural regions at 2.4GHz. We defined three scenarios tied to rural regions and we conducted a measurement campaign. We compared five selected models and we found that Liechty model is the more precise one. But Liechty model outperforms others models in separated environments. Further, we improved this model in combined environment (considering LOS and NLOS) by considering the distance to the first breakpoint. We obtained a better prediction model with a mean error of $2.33 \mathrm{~dB} / 2.67 \mathrm{~dB}$ and a standard deviation of $4.02 \mathrm{~dB} / 4.21 \mathrm{~dB}$ in worst case in wooded area and a mean error of $2.36 \mathrm{~dB} / 2.61 \mathrm{~dB}$ and a standard deviation of $4.01 \mathrm{~dB} / 4.19 \mathrm{~dB}$ in worst case in built space, respectively for $300 \mathrm{Mbps} / 150 \mathrm{Mbps}$ USB receivers. A nonneglecting observation is the fact that standard deviation and the mean error in path loss prediction increase for receiver with low data rate. It would be interesting to study this variation in other to propose more realistic models.

Since we usually observe more than one breakpoint between the transmitter and the receiver, it can be interesting to study the impact of other breakpoints on the precision of the path loss model and to consider those points without making the model more complex. A last perspective is to implement the path loss model in network simulator in order to predict more accurately the signal path loss in likewise rural regions.

\section{REFERENCES}

[1]. Fendji, Ebongue Kedieng, J.L.: Rethinking Network Connectivity in Rural Communities in Cameroon. Presented at the May 17 (2015).

[2]. Isong, B., Dladlu, N., Magogodi, T.: Computer Network and Information Security. Comput. Netw. Inf. Secur. 11, 14-22 (2016).

[3]. Fendji Kedieng Ebongue, J.L., Nlong, J.M.: Rural Wireless Mesh Network: A Design Methodology. Int. J. Commun. Netw. Syst. Sci. 8, 1-9 (2015).

[4]. Egli, J.: Radio Propagation above 40 MC over Irregular Terrain. Proc. IRE. 45, 1383-1391 (1957).

[5]. OKUMURA, Y.: Field strength and its variability in VHF and UHF land-mobile radio service. Rev. Electr. Commun. Lab. 16, 825-873 (1968).

[6]. Hata, M.: Empirical formula for propagation loss in land mobile radio services. IEEE Trans. Veh. Technol. 29, 317-325 (1980).

[7]. Erceg, V., Greenstein, L.J., Tjandra, S., Parkoff, S.R., Gupta, A., Kulic, B., Julius, A., Jastrzab, R.: An empirically-based path loss model for wireless channels in suburban environments. In: IEEE GLOBECOM 1998 (Cat. NO. 98CH36250). pp. 922-927. IEEE.

[8]. Medeisis, A., Kajackas, A.: On the use of the universal Okumura-Hata propagation prediction model in rural areas. In: VTC2000-Spring. 2000 IEEE 51st Vehicular Technology Conference Proceedings (Cat. No.00CH37026). pp. 1815-1818. IEEE. 
Jean Louis Ebongue Kedieng Fendji, Nelson Maguelva Mafai, Jean Michel Nlong; Slope-based Empirical Path Loss Prediction Models for Rural Networks at $2.4 \mathrm{GHz}$, Transactions on Networks and Communications, Volume 7 No. 1, February (2019); pp: 84-101

[9]. Rakesh, J., A., W.V., Dalal, U.: A Survey of Mobile WiMAX IEEE 802.16m Standard. (2010).

[10]. Akl, R.G., Tummala, D., Li, X.: Indoor Propagation Modeling at 2.4 GHZ for IEEE 802.11 Networks. Sixth Int. Assoc. Sci. Technol. Dev. Int. Multi-Conference Wirel. Opt. Commun. 2006, Banff, Alberta, Canada. (2006).

[11]. Liechty, L.C.: Path Loss Measurements and Model Analysis of a $2.4 \mathrm{GHz}$ Wireless Network in an Outdoor Environment. (2007).

[12]. Yenke, B.O., Tala, D.C.M., Louis, J., Fendji, E.K.: Extended Probabilistic Cost Model (EPCM): A Framework for Cost Estimation of Wireless Network Deployment in Rural Areas. Inf. Eng. Electron. Bus. 1, 1-9 (2017).

[13]. Fendji Kedieng Ebongue, J.L., Mafai, N., Nlong, J.M., Ebongue, J.L.F.K., Nelson, M., Nlong, J.M.: Empirical path loss models for $802.11 \mathrm{n}$ wireless networks at $2.4 \mathrm{GHz}$ in rural regions. In: Lecture Notes of the Institute for Computer Sciences, Social-Informatics and Telecommunications Engineering, LNICST. pp. 53-63 (2015).

[14]. Tan, S.Y., Tan, H.S.: Propagation model for microcellular communications applied to path loss measurements in Ottawa city streets. IEEE Trans. Veh. Technol. 44, 313-317 (1995).

[15]. Sun, Q., Tan, S.Y., Teh, K.C.: Analytical Formulae for Path Loss Prediction in Urban Street Grid Microcellular Environments. IEEE Trans. Veh. Technol. 54, 1251-1258 (2005).

[16]. Taflove, A., Hagness, S.C.: Computational electrodynamics : the finite-difference time-domain method. Artech House (2005).

[17]. Rana, M.M., Mohan, A.S.: Segmented-Locally-One-Dimensional-FDTD Method for EM Propagation Inside Large Complex Tunnel Environments. IEEE Trans. Magn. 48, 223-226 (2012).

[18]. Friis, H.T.: A Note on a Simple Transmission Formula. Proc. IRE. 34, 254-256 (1946).

[19]. Seidel, S.Y., Rappaport, T.S.: $914 \mathrm{MHz}$ path loss prediction models for indoor wireless communications in multifloored buildings. IEEE Trans. Antennas Propag. 40, 207-217 (1992).

[20]. Andrade, C.B., Hoefel, R.P.F.: IEEE 802.11 WLANs: A comparison on indoor coverage models. In: CCECE 2010. pp. 1-6. IEEE (2010).

[21]. Cichon, D.J., Kürner, T.: Digital mobile radio towards future generation systems: Cost 231 final report. COST Eur. Coop. F. Sci. Tech. Res. 231, (1993).

[22]. Green, D.B., Obaidat, A.S.: An accurate line of sight propagation performance model for ad-hoc 802.11 wireless LAN (WLAN) devices. In: 2002 IEEE International Conference on Communications. Conference Proceedings. ICC 2002 (Cat. No.02CH37333). pp. 3424-3428. IEEE. 
[23]. ERCEG, V.: Channel Models for Fixed Wireless Applications. IEEE 802.16.3c-01/29rl. (2001).

[24]. Brown, D. and M. S. Gregory: A report on technology independent methodology for the modelling, simulation, and empirical verification of wireless communication system performance in noise and interference limited systems operation on frequencies between 30 and1500 mhz. Technical report, Telecommunication Industry Association (TIA) TR8 Working Group 8.8, May 1997.

[25]. National Institute of Standards and Technology: Hata and CCIR Formulas IST. http://w3.antd.nist.gov/wctg/manet/calcmodels_r1.pdf, visited: March 2018.

[26]. European Radio Communications Committee: The analysis of the coexistence of FWS cells in the 3.4 - 3.8 GHz bands. ERC Report, European Conference of Postal and Telecommunications Administrations (CEPT), May 2003. 